\title{
Response of extreme haloarchaeon Haloarcula argentinensis RR10 to simulated microgravity in clinorotation
}

\author{
Rebecca Thombre $^{1} \cdot$ Vinaya Shinde $^{1} \cdot$ Jyotsana $_{\text {Dixit }^{2}} \cdot$ Sagar Jagtap $^{3} \cdot$ \\ Pandit B. Vidyasagar ${ }^{4}$
}

Received: 19 July 2016/ Accepted: 23 December 2016/Published online: 11 April 2017

(c) The Author(s) 2017. This article is an open access publication

\begin{abstract}
Gravity is the fundamental force that may have operated during the evolution of life on Earth. It is thus important to understand as to what the effects of gravity are on cellular life. The studies related to effect of microgravity on cells may provide greater insights in understanding of how the physical force of gravity shaped life on Earth. The present study focuses on a unique group of organisms called the Haloarchaea, which are known for their extreme resistance to survive in stress-induced environments. The aim of the present investigation was to study the effect of simulated microgravity on physiological response of extremely halophilic archaeon, Haloarcula argentinensis RR10, under slow clinorotation. The growth kinetics of the archaeon in microgravity was studied using the Baryani model and the viable and apoptotic cells were assessed using propidium iodide fluorescent microscopic studies. The physiological mechanism of adaptation was activation of 'salt-in' strategy by intracellular sequestration of sodium ions as detected by EDAX. The organism upregulated the production of ribosomal proteins in simulated microgravity as evidenced by Matrix-assisted laser desorption ionization Time of flight-Mass
\end{abstract}

Rebecca Thombre

rebecca.thombre@gmail.com

1 Department of Biotechnology, Modern College of Arts, Science and Commerce, Shivajinagar, Pune, Maharashtra 411005, India

2 School of Basic Medical Sciences, Savitribai Phule Pune University, Ganeshkhind, Pune, Maharashtra 411007, India

3 Department of Physics, H. V. Desai College, Pune, Maharashtra 411002, India

4 Swami Ramanand Teerth Marathwada University, Nanded, Maharashtra 431606, India
Spectrophotometry. Simulated microgravity altered the antibiotic susceptibility of the haloarchaeon and it developed resistance to Augmentin, Norfloxacin, Tobramycin and Cefoperazone, rendering it a multidrug resistant strain. The presence of antibiotic efflux pump was detected in the haloarchaeon and it also enhanced production of protective carotenoid pigment in simulated microgravity. The present study is presumably the first report of physiological response of $H$. argentinensis RR10 in microgravity simulated under slow clinorotation.

Keywords Simulated microgravity · Haloarchaea · Haloarcula argentinensis . 'Salt-in' strategy .

Antimicrobial resistance $\cdot$ Physiological mechanisms . Growth kinetics

\section{Introduction}

Outer space and planets, excluding Earth, has an environment that is characterized by harsh inhabitable conditions. The profound disparity in gravity, radiation, pressure, atmosphere, oxygen, salinity and nutrients are responsible for the hostile and extreme conditions pernicious for growth of microbial life in outer space. One of the contrasting differences in the environment of Earth as compared to the environment existing in spacecraft, spaceflights and international space stations located in outer space is dissimilitude in gravitational forces. The gravity in these space locations is diminished from normal gravity (NG) of $1 \mathrm{~g}$ (gravitational force of Earth) to $10^{-2}$ to $10^{-6} \mathrm{~g}$ called as microgravity. Microgravity is responsible for the experience of weightlessness in spaceflights and spacecrafts and recently studies related to the effect of microgravity on cells have garnered profound significance. 
Effect of microgravity can be studied in international space stations or the microgravity conditions can be simulated in a laboratory. The essential change in environment observed in spaceflights and spacecraft is the change in the gravitational force. In spaceflights and international space stations, the gravitational force is diminished to such an extent that objects appear weightless as a consequence of which astronauts and objects float inside the spacecraft. Though the gravitational force doesn't become zero, it is reduced to small amount $\left(10^{-6} \mathrm{~g}\right)$ and thus is called as microgravity.

Study of microgravity in the laboratory can be performed by simulation of microgravity in specialized bioreactors that have low shear dynamics and such an environment is called as low-shear modeled microgravity (LSMMG) environment. Specialized rotating ground based bioreactors similar to suspension culture vessels used in tissue culture are utilized for generation of LSMMG and these are known as high-aspect-ratio vessel (HARV) (Purevdorj-Gage et al. 2006). A similar device called as a Rotary Cell Culture System manufactured by Synthecon (Houston, TX) has been used to simulate microgravity (Xu et al. 2015). The effect of microgravity on cells can also be studied using a clinostat that rotates at a low speed along a horizontal axis so as to negate the forces of gravity and simulate a microgravity environment (Jagtap et al. 2011).

A clinostat is a device that can be used to simulate microgravity conditions that exist in outer space or spaceflights for microbial suspension cell cultures (Klaus et al. 1997). The simulated microgravity is created by rotation of a cylinder at constant velocity completely filled with a liquid broth medium containing cells devoid of any air bubbles or spaces (Klaus et al. 1998).

These studies have been carried out in the clinostat at slow rotation (Jagtap et al. 2011) (ranging from 0.25 to $3 \mathrm{rpm}$ ) and at higher rotations (Luna et al. 2015) (30-75 rpm). When the clinostat is operated at proper rotation, the cells neither sediment nor adhere to the container wall thereby reducing the relative fluid motion and simulating weightlessness as experienced in spaceflights. The term "simulated microgravity" can thus be defined as a state resultant of clinorotation, in which the force due to gravity $(g)$ is not completely obliterated rather the rotation at a constant velocity results in the $g$-vector being timeaveraged to near-zero (Klaus et al. 1997, 1998). The response of haloarchaea Haloferax mediterranei DSM $1411^{\mathrm{T}}$ and Halococcus dombrowskii DSM $14522^{\mathrm{T}}$ to microgravity simulated in RCCS, Synthecon has been previously investigated (Dornmayr-Pfaffenhuemer et al. 2011). However, there are no studies related to the response of haloarchaea in clinorotation.

In the present study, a clinostat under slow rotation was used for studying the effect of simulated microgravity (SMG) on halophilic archaea. Halophiles are considered as model organisms for stress related experiments and astrobiology related investigations (DasSarma 2006). Haloarchaea are halophilic archaea that have a mandatory requirement of $1.5 \mathrm{~mol}^{-1} \mathrm{NaCl}$ for growth and extreme haloarchaea can survive up to $5.1 \mathrm{~mol}^{-1} \mathrm{NaCl}$ (Thombre et al. 2016a). They have the inherent and acquired ability to survive and adjust in manifold stresses which is the key factor for studies related to astrobiology. Haloarchaea have also been known to survive in halite crystals for long periods and survival ability was of paramount importance in application of survival studies of haloarchaea in outer space environments like the European Space Agency's, Biopan facility and International Space Station (Mancinelli et al. 1998; Stan-Lotter et al. 2002; Fendrihan et al. 2006). The studies related to survival of haloarchaea in microgravity are crucial in contributing valuable information regarding exploration of life in outer space. Besides these organisms, spores and coccoid cells are considered as 'micronauts' that are microorganisms that can survive travel from one planet to another (Dornmayr-Pfaffenhuemer et al. 2011). The survival mechanisms of haloarchaea during stress-conditions may provide useful insights to the possibility of their survival elsewhere.

The present study focuses on studying the growth kinetics of extremely halophilic archaeon, Haloarcula argentinensis RR10 in simulated microgravity and assessing the adaptation response of the organisms under clinorotation. The haloarchaeon utilizes a combination of physiological responses including intracellular sequestration of sodium ions, production of stress proteins and counteraction of cellular damage due to oxidative stress balanced by production of pigment to adapt to simulated microgravity. The response and plausible mechanism of survival and adaptation of haloarchaea during simulated microgravity is being reported presumably for the first time in $H$. argentinensis RR10.

\section{Materials and methods}

\section{Growth of microorganism}

The organism used in the present study was haloarchaeon, $H$. argentinensis strain RR10 (GenBank/EMBL/DDBJ accession number KP712898, MCC 2923) isolated in our laboratory previously from salterns of Mumbai, India (Thombre et al. 2016b). It is an extreme halophile that requires $3-5$ mol $1^{-1}$ sodium chloride for growth. It was cultured in broth culture in an orbital shaker at $40{ }^{\circ} \mathrm{C}$ at $100 \mathrm{rev}^{\mathrm{min}}{ }^{-1}$ in Sehgal and Gibbons (SG) medium containing (g/L) casamino acids (7.5), yeast extract (10), potassium chloride (2), trisodium citrate (3), magnesium 
sulfate (20) and pH- 7.5 supplemented with $4.28 \mathrm{~mol} \mathrm{l}^{-1}$ sodium chloride (Sehgal and Gibbons 1960).

\section{Growth kinetic studies of Haloarcula argentinensis RR10 during simulated microgravity generated in clinostat}

The growth kinetics of $H$. argentinensis RR10 under simulated microgravity (SMG) and normal gravity (NG) was studied by exposing the organism to SMG in a clinostat. The microgravity conditions were simulated in a 1-D clinostat developed in-house in the Department of Physics, S.P. Pune University, India (Jagtap et al. 2011). Exponential phase culture of $H$. argentinensis RR10 $\left(\sim 10^{6} \mathrm{CFU} / \mathrm{ml}\right)$ in $\mathrm{SG}$ medium was dispensed in $50 \mathrm{ml}$ sterile cylindrical syringes and rotated in horizontal axis generating simulated microgravity of $8.94 \times 10^{-5} \times g$ at $40{ }^{\circ} \mathrm{C}$. The control consisted of cells of $\mathrm{H}$. argentinensis RR10 in $50 \mathrm{ml}$ sterile syringe maintained in normal gravity (NG) conditions $(1 \times g)$ at $40{ }^{\circ} \mathrm{C}$ without agitation. Samples were withdrawn aseptically every $24 \mathrm{~h}$ from the medium maintained in SMG and NG conditions and the growth was measured in terms of absorbance at $600 \mathrm{~nm}$ using UV Visible Spectrophotometer (UV-1800, Shimadzu, Japan). The growth kinetics were studied using the Baranyi model (Baranyi and Roberts 1994) and the curve-fitting DMFit programme and the growth rate $(\mu)$, generation time $(g)$ and the specific growth rate constant $(k)$ and $\lambda$ (lag phase) were calculated from the growth curve as described earlier (Salgaonkar et al. 2016; Metris et al. 2006; Robinson et al. 2005; Thombre et al. 2016c).

\section{Assessment of viability of haloarchaea in SMG using propidium iodide fluorescent staining}

The effect of SMG on viability of haloarchaea was studied using propidium iodide staining and visualization using fluorescence microscopy. Propidium iodide stains the cells that exhibit damaged membrane red in color indicating non-viable, metabolically injured or necrotic cells. Propidium iodide cannot penetrate intact membranes of live cells, hence viable cells don't take up the red color (StanLotter et al. 2002). Briefly, the cells exposed to SMG and NG were stained with propidium iodide solution (Sigma, Germany) and observed under Fluorescent microscope (AV 10-Zeiss, Germany) with Apotome and $400 \mathrm{X}$ filter using the Axiovision software for imaging and the number of damaged cells (cells stained red with propidium iodide) were counted (Rieger et al. 2011). The percentage of viable cells was calculated as: $\%$ viability $=$ (number of viable cells counted/total number of cells counted $) \times 100$.
Study of 'salt-in strategy' of osmoadaptation in haloarchaea during SMG by energy dispersive $\mathrm{X}$-ray spectroscopy (EDAX) analysis

On exposure to simulated microgravity, haloarchaea accumulate intracellular ions as a part of the 'salt-in strategy' of adaptation. The archaeal intracellular ion accumulation in response to SMG was studied using EDAX analysis (Jeol, Japan). The sample preparation of $H$. argentinensis RR10 cells exposed to simulated microgravity and normal gravity for EDAX was as detailed previously (Thombre et al. 2016b).

\section{Production of protective carotenoid pigments produced in response to SMG}

The production of protective red carotenoid pigment produced by $H$. argentinensis RR10 in response to SMG and NG was studied. The total pigment content present was estimated by measuring the absorbance of the culture broth at $490 \mathrm{~nm}$ ( $\lambda_{\max }$ of haloarchaeal carotenoid) using UV-Vis Spectrophotometer (UV-1800, Shimadzu, Japan) (Abbes et al. 2013).

Effect of SMG on antibiotic resistance of $\boldsymbol{H}$. argentinensis RR10 and detection of antibiotic efflux pumps

The changes in the antibiotic resistance pattern of $H$. argentinensis RR10 in response to SMG and NG were studied by disc diffusion method as per Clinical and Laboratory Standards Institute (CLSI) guidelines (2012) and Shinde and Thombre (2016). Briefly, $100 \mu$ of the culture (absorbance corresponding to $0.5 \mathrm{Mc}$ Farland standard) exposed to SMG and NG (7th day) was spread on SG medium and antibiotic discs of Augmentin, Norfloxacin, Nalidixic acid, Imipenem, Tobramycin, Cefoxitin, Cefoperazone and Piperacillin/Tazobactam (HiMedia, India) were placed aseptically on it and incubated for 7-10 days at $40{ }^{\circ} \mathrm{C}$. The results were interpreted as resistant or sensitive using interpretive criteria according to the CLSI guidelines (CLSI 2014). Detection of antibiotic resistance efflux pumps responsible for antibiotic resistance was performed by slightly modified ethidium bromide-agar cartwheel method (Martins et al. 2011; Shinde and Thombre 2016). Cells of $H$. argentinensis RR10 (absorbance adjusted to 0.5 of Mc Farland standard) exposed to SMG and NG were streaked on SG agar supplemented with $4.8 \mathrm{~mol} \mathrm{l}^{-1}$ sodium chloride containing $\mathrm{EtBr}$ $\left(0-2.5 \mathrm{mg} \mathrm{L}^{-1}\right)$ divided into radial sectors and incubated at $40{ }^{\circ} \mathrm{C}$ for $5-7$ days till growth appeared. The plates were examined under UV trans illuminator and the minimum 
concentration of $\mathrm{EtBr}\left(\mathrm{MIC}_{\mathrm{EtBr}}\right)$ that produced fluorescence was recorded.

\section{SDS-PAGE of differential proteins expressed in haloarchaea during SMG}

After exposure to simulated microgravity, the proteins expressed in cytosol of $H$. argentinensis RR10 were subjected to separation using 1-D SDS PAGE as described previously (Thombre et al. 2016b; Otte et al. 2014). The protein bands obtained after staining from the cells exposed to SMG and NG were compared and the differentially expressed protein bands in SMG sample were identified and selected for peptide mass finger printing. The selected bands were excised from the gel and trypsinized as described by Otte et al. (2014). Briefly, the protein sample was trypsinized with trypsin digestion buffer $(50 \mathrm{mM}$ $\mathrm{NH}_{4} \mathrm{HCO}_{3}, 5 \mathrm{mM} \mathrm{CaCl} 2,12.5 \mathrm{ng} / \mu \mathrm{l}$ trypsin) and the peptide digest extract was prepared as described earlier (Thombre et al. 2016b).

\section{Identification of differential proteins using MALDI TOF-MS and MASCOT analysis}

The peptide digest extracted from the gel piece $(1 \mu \mathrm{l})$ was premixed with equal volume of matrix and spotted on a matrix-assisted laser desorption ionization (MALDI) plate as per manufacturer's instructions. The Peptide mass fingerprint (PMF) was obtained using MALDI TOF-mass spectrometer (Ultraflex II, Bruker Daltonics, Germany) in the reflector mode. The data generated was analyzed using Swiss-Prot database using MASCOT search engine(Matrix Science, London, United Kingdom) with a peptide mass tolerance of 100 ppm (Trotter et al. 2015; Govekar et al. 2012; Thombre et al. 2016b).

\section{Statistical Analysis}

All experiments were performed in triplicates. Statistical analysis was performed using Excel 2016. All values were expressed as the mean \pm standard error (SE). Statistical significance between groups was calculated using the paired Student's $t$ test and the value of $p<0.10$ was considered to be statistically significant.

\section{Results}

\section{Growth kinetic studies of Haloarchaea in simulated microgravity generated in clinostat}

The growth kinetics of $H$. argentinensis RR10 in simulated microgravity under clinorotation was studied. Under the effect of simulated microgravity, the growth was faster as compared to the same culture incubated under normal gravity conditions (Fig. 1). The difference in growth between normal and simulated microgravity was statistically significant $(t$ value $=1.4028$ and $p$ value $=0.095475$ at $p<0.10)$ and growth was higher in simulated microgravity. The growth kinetics in simulated microgravity and normal gravity were studied using the growth curve plots and no lag phase $(\lambda)$ was observed in both conditions. The doubling time or generation time $(t)$ was 8 and $20 \mathrm{~h}$ and growth rate constant $(k)$ was 0.0866 and $0.03465 \mathrm{~h}^{-1}$ for simulated and normal gravity respectively. The Monod's maximum specific growth rate was also calculated using the Baryani model and DMFit curve-fitting programme and the growth rate $(\mu)$ was calculated as 0.12 and $0.08 \mathrm{~h}^{-1}$ for simulated microgravity and normal gravity, respectively. It is apparent from the growth kinetic studies that the haloarchaeon demonstrates maximum growth in simulated microgravity. The viability of the cells exposed to altered gravity was assessed using fluorescent microscopy using propidium iodide. Propidium iodide is impermeable to viable cells that maintain cell membrane integrity. However, due to stress, cells undergo necrosis and exhibit damaged cell membrane due to loss of membrane integrity as a result of which the propidium iodide is taken up by the cells and it demonstrates red fluorescence in non viable cells. When haloarchaea were exposed to simulated microgravity, they demonstrated faster growth and more number of viable cells as compared to haloarchaea cultured under normal gravity (Fig. 2). The number of non viable cells demonstrating red fluorescence was significantly higher under normal gravity in comparison to the cells exposed to SMG (Fig. 3). Besides, it was observed that cellular aggregation under SMG was higher and clumping

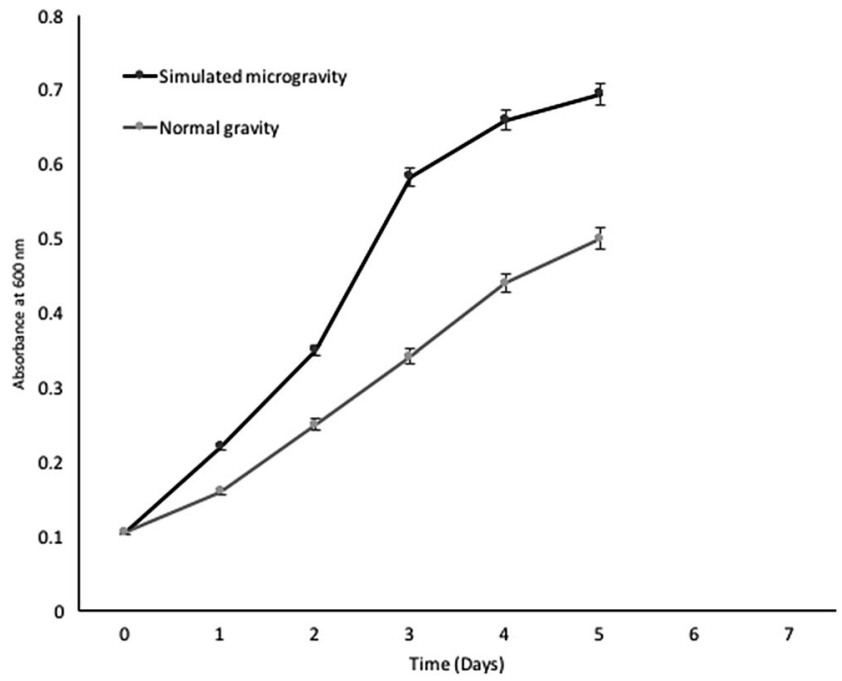

Fig. 1 Effect of simulated microgravity and normal gravity on growth of Haloarcula argentinensis RR10 
Fig. 2 a Fluorescence images of Haloarcula argentinensis RR10 exposed to normal gravity stained by propidium iodide. b Fluorescence images of Haloarcula argentinensis RR10 exposed to simulated microgravity stained by propidium iodide
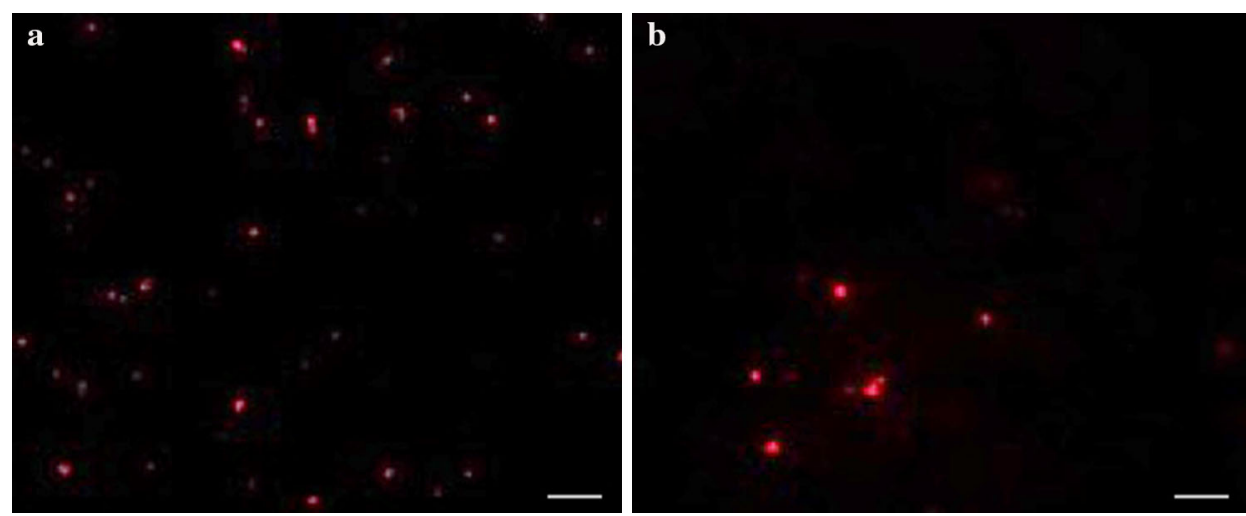

of cells was observed in simulated microgravity conditions as seen in Fig. 2b. However, under normal gravity conditions, lesser aggregation was seen as evidenced by the smaller size of cells in NG (Fig. 2a). It is suggested that further studies using commercially available Live-Dead staining kits and FACS could provide deeper insights about viability of cells and the presence of dead, damaged and viable but unculturable cells in simulated microgravity and normal gravity.

\section{Study of 'salt-in strategy' of osmoadaptation in haloarchaea during SMG by energy dispersive $\mathrm{X}$-ray spectroscopy (EDAX) analysis}

On exposure to harsh conditions, haloarchaea utilize the 'salt-in strategy' to counteract the effect of osmotic stress. Using this strategy, the organism sequesters salt intracellularly to counter balance the gradients of salts created during stress. In order to study if this strategy is utilized by $H$. argentinensis RR10 during simulated microgravity, a detection of the intracellular archaeal ions sequestered in simulated microgravity and normal gravity was performed. It was observed that in simulated microgravity, the haloarchaeon increased the sequestration sodium, potassium and chloride ions significantly (Fig. 4). Maximum sequestration during simulated microgravity was of sodium ions and the trend in ion accumulation in SMG was $\mathrm{Na}>\mathrm{Cl}>\mathrm{K}$ and for $\mathrm{NG}$ is $\mathrm{Cl}>\mathrm{Mg}>\mathrm{Na}>\mathrm{K}$.

\section{Production of protective carotenoid pigments produced in response to simulated microgravity}

Red-orange colored pigments are produced by haloarchaea to tackle oxidative stress caused by external environmental factors affecting the culture during growth. It was observed that when $H$. argentinensis RR10 was exposed to SMG, it produced significantly higher amount of deep red-orange pigmentation in comparison to its pigment production in

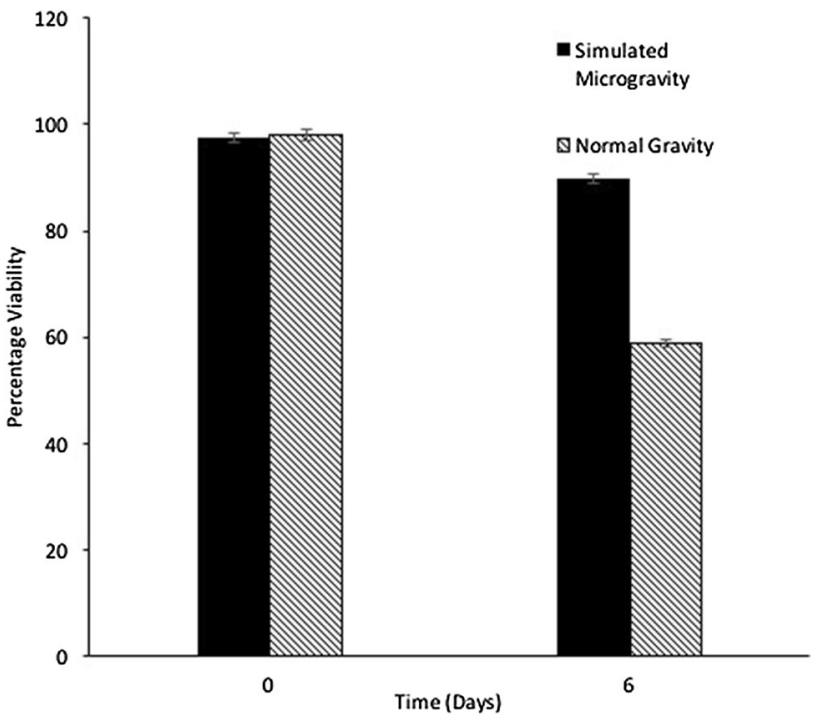

Fig. 3 Percentage viability of haloarchaeal cells exposed to simulated microgravity and normal gravity detected by propidium iodide staining

normal gravity (Fig. 5). The UV-Vis spectral scan and absorption maxima of the pigment indicates that the carotenoid produced resembles the pigment bacterioruberin (Abbes et al. 2013).

\section{Effect of SMG on antibiotic resistance of $\boldsymbol{H}$. argentinensis RR10 and detection of antibiotic efflux pumps}

The wild type strain of $H$. argentinensis RR10 is sensitive to the antibiotics Augmentin, Norfloxacin, Imipenem, Tobramycin, Cefoxitin, Cefoperazone and Piperacillin/ Tazobactam and resistant to Nalidixic acid under normal gravity conditions. On exposure to simulated microgravity, $H$. argentinensis RR10 developed resistance to many antibiotics as depicted in Table 1. As the isolate demonstrated change in its antibiotic resistance after exposure to SMG, the possible involvement of antibiotic efflux pump 
Fig. 4 Relative percentage of intracellular archaeal ion accumulation by Haloarcula argentinensis RR10 when exposed to simulated microgravity and normal gravity detected by EDAX
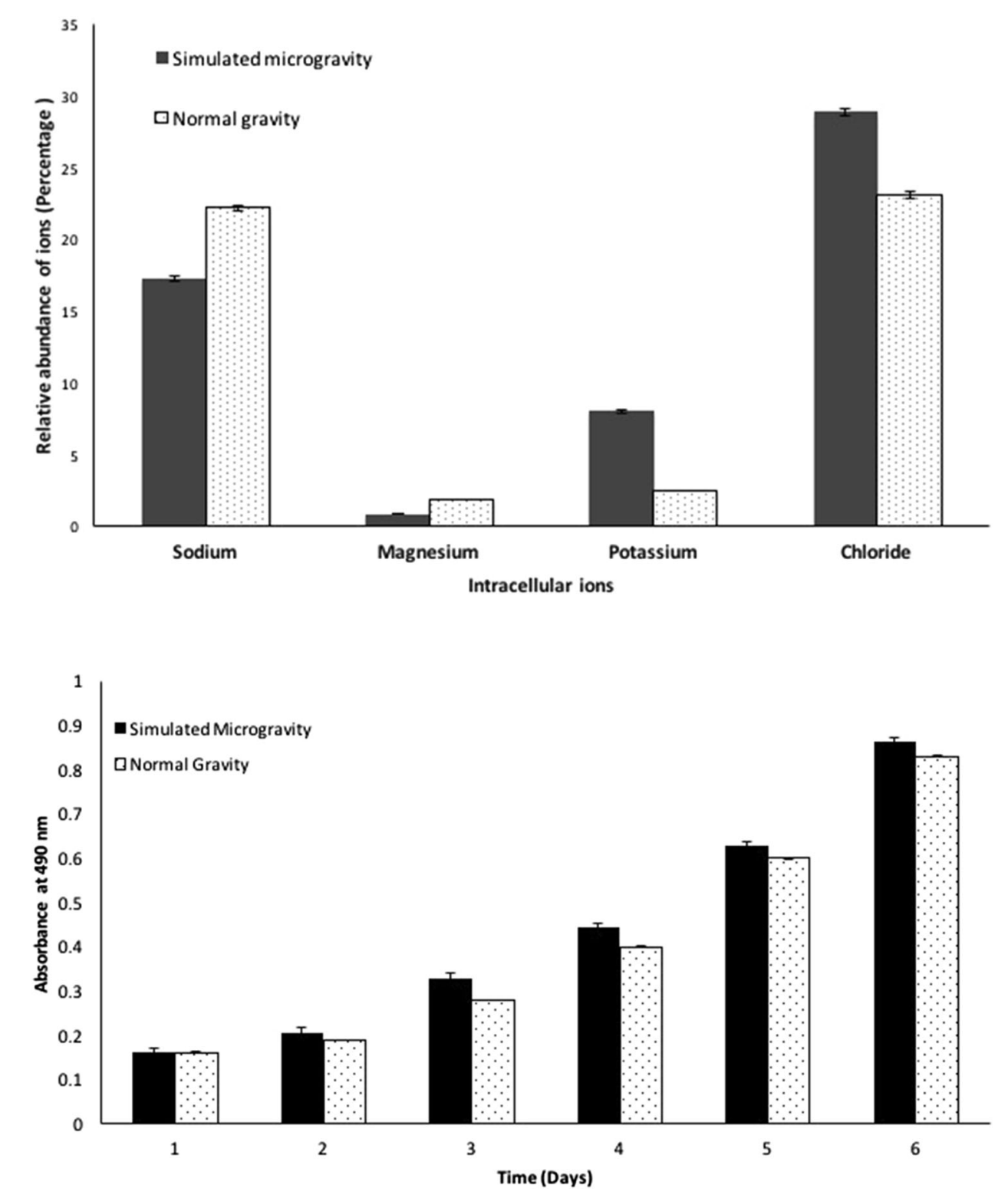

Fig. 5 Effect of simulated microgravity and normal gravity on total carotenoid pigment production by Haloarcula argentinensis RR10 was studied by modified ethidium bromide cartwheel assay. It was observed that cells of $H$. argentinensis RR10 showed the upregulation of efflux pump activity in SMG conditions as evidenced by visualization of fluorescence under UV-trans illuminator. The minimum concentration of $\mathrm{EtBr}\left(\mathrm{MIC}_{\mathrm{EtBr}}\right)$ that produced fluorescence of the haloarchaeal culture when exposed to normal gravity was $2 \mathrm{mg} \mathrm{\textrm {L } ^ { - 1 }}$. However, on exposure to simulated microgravity, the efflux pump activity was seen in the cartwheel assay at a much lower concentration of $\mathrm{MIC}_{\mathrm{EtBr}}=1.5 \mathrm{mg} \mathrm{L} \mathrm{L}^{-1}$. There was increase in the antibiotic resistance of the archaeon on exposure to simulated microgravity and the organism responded by over expressing the efflux pumps. The fluorescence produced by the cells when exposed to simulated microgravity was greater as compared to the cells cultured in normal gravity as observed by the ethidium bromide cartwheel assay.

\section{Differential proteins expressed in response to SMG in $H$. argentinensis RR10}

We examined the effects of simulated gravity conditions on $H$. argentinensis at protein level using a non targeted proteomic 1D-DIGE approach and MALDI TOF analysis. Five differential bands were identified using MS followed by MASCOT analysis (Table 2). Majority of the peptides identified in response to simulated microgravity were ribosomal proteins ranging in the size $10-17 \mathrm{kDa}$. The abundance of ribosomal proteins unregulated was a significant finding in terms of response to simulated microgravity. 
Table 1 The antibiotic resistance profile of Haloarcula argentinensis RR10 exposed to Simulated microgravity and normal gravity conditions

\begin{tabular}{|c|c|c|c|c|c|c|c|c|}
\hline \multirow[t]{2}{*}{ S. no. } & \multirow[t]{2}{*}{ Antibiotic } & \multirow[t]{2}{*}{$\begin{array}{l}\text { Concentration } \\
(\mu \mathrm{g})\end{array}$} & \multirow[t]{2}{*}{ Class/type of antibiotic } & \multicolumn{3}{|c|}{$\begin{array}{l}\text { Interpretive criteria } \\
\text { for zone diameter }{ }^{\mathrm{a}}(\mathrm{mm})\end{array}$} & \multicolumn{2}{|c|}{$\begin{array}{l}\text { Antibiotic resistance profile of } \\
\text { Haloarcula argentinensis } \mathrm{RR} 1 \mathrm{C}\end{array}$} \\
\hline & & & & $S$ & I & $\mathrm{R}$ & Normal gravity & $\begin{array}{l}\text { Simulated } \\
\text { microgravity }\end{array}$ \\
\hline 1 & Augmentin & 30 & Penicillin + clavulanic acid & - & - & - & $S$ & $\mathrm{R}$ \\
\hline 2 & Norfloxacin & 10 & Fluoroquinolone & $\geq 17$ & $13-16$ & $12 \leq$ & $S$ & $\mathrm{R}$ \\
\hline 3 & Nalidixic acid & 30 & Quinolone & $\geq 19$ & $14-18$ & $13 \leq$ & $\mathrm{R}$ & $\mathrm{R}$ \\
\hline 4 & Imipenem & 10 & Carbapenem & $\geq 23$ & $20-22$ & $19 \leq$ & $S$ & $S$ \\
\hline 5 & Tobramycin & 10 & Aminoglycoside & $\geq 15$ & $13-14$ & $12 \leq$ & $S$ & $\mathrm{R}$ \\
\hline 6 & Cefoperazone & 75 & Cephalosporin & $\geq 21$ & $16-20$ & $15 \leq$ & S & $\mathrm{R}$ \\
\hline 7 & Cefoxitin & 30 & Cephamycin & $\geq 18$ & $15-17$ & $14 \leq$ & $S$ & S \\
\hline 8 & Piperacillin/tazobactam & $100 / 10$ & $\beta$-lactam $+\beta$-lactamase inhibitor & $\geq 21$ & $18-20$ & $17 \leq$ & $\mathrm{S}$ & $\mathrm{S}$ \\
\hline
\end{tabular}

$R$ resistant, $I$ intermediate, $S$ sensitive, $n d$ not determined

${ }^{a}$ Interpretive criteria of Gram-negative bacteria as per guidelines of CLSI M100-S24 for disk diffusion

Table 2 Differential proteins expressed by Haloarcula argentinensis RR10 in SMG studied using peptide mass fingerprinting (PMF)

\begin{tabular}{lllcc}
\hline S. no. & Description of putative peptide/protein & Gene & Putative pI & Putative molecular weight (Da) \\
\hline 1 & 50S ribosomal protein L22 & rpl22 & 5.0 & $17,191.480$ \\
2 & 30S ribosomal protein S8 & rps8 & 7.7 & $14,305.200$ \\
3 & Translation initiation factor 2 subunit beta & eif2b & 8.9 & $16,215.180$ \\
4 & 30S ribosomal protein S11 & rps11 & 11.6 & $14,082.540$ \\
5 & 50S ribosomal protein L31e & rpl31e & 4.8 & $10,129.080$ \\
\hline
\end{tabular}

\section{Discussion}

Understanding the evolution of life-forms on Earth and their survival during initial extremely unfavorable environmental conditions is of paramount importance. With the progression of life, the inherent and acquired abilities to survive in multiple stresses especially altered gravity may have been a key factor for survival of organisms. Haloarchaea are a unique group of poly-extremophilic organisms with unusual features and are known to survive multiple stresses. Their physiological robustness and ability to survive in the presence of steep fluctuations in temperature, atmosphere, oxygen, radiations, salinity, perchlorate, epsomite, gravity and numerous other stresses has always intrigued scientists. They are apt model exo-philes to study possible effect and adaptability of life forms in spacecraft, in altered gravity like microgravity and hypergravity, as well as in outer space (Thombre et al. 2017). Currently, studies aim at understanding the effect of changes in mechanical forces that occur in microgravity and other low-shear environments on different microbial parameters (Nickerson et al. 2004). Microgravity is known to affect the physiology of cells, gene expression and increase the antibiotic resistance of microorganisms (Xu et al. 2015).
Previously studies have been conducted on the effect of space like conditions including microgravity on growth of Escherichia coli (Xu et al. 2015), Salmonella typhimurium (Wilson et al. 2007), Saccharomyces cerevisiae (PurevdorjGage et al. 2006) and plants (Jagtap et al. 2011). The effect of simulated microgravity in clinorotation have been studied using E. coli (Brown et al. 2002; Arunasri et al. 2013) and the fungi Aspergillus niger and Candida albicans (Yamazaki et al. 2012). However, this is the first report on effect of simulated microgravity on extremely halophilic archaeon $H$. argentinensis RR10 in clinorotation. This strain was isolated from a harsh hypersaline thalossohaline salterns of Mumbai, India. This haloarchaeon was selected for the present study as it is a polyextremophile and it demonstrated survival over a wide range of salinity, magnesium concentration, temperature and $\mathrm{pH}$.

On exposure to simulated microgravity, the haloarchaeon $H$. argentinensis RR10 still continued to demonstrate faster growth as evidenced by increase in absorbance, lesser doubling time and higher percentage viability observed by fluorescence microscopy in comparison to the cells cultured in normal gravity. E. coli when exposed to simulated microgravity showed a similar statistically 
significant increase in growth as compared to $E$. coli incubated in NG (Xu et al. 2015). The exact responses and adaptations that haloarchaea elicit in stresses and such altered gravity environment is still unidentified. The general strategies these organisms utilize in ionic stresses are the 'salt-in strategy' and the 'organic osmolyte strategy' also referred to as compatible solute strategy. In the organic osmolyte strategy, haloarchaea synthesize or carry out the uptake of organic compatible solutes in the cytosol. Thus, the osmotic potential is counter balanced despite maintenance of low salt intracellularly. The compatible solutes are ectoine, glycine betaine, trehalose, sorbitol, glycerol and amino acid derivatives. This strategy is more common in halophilic bacteria. The 'salt-in strategy' is employed by true halophiles, including halophilic archaea and extreme haloarchaea (Shivanand and Mugeraya 2011). In this mechanism the organism selectively sequesters the cations (sodium or potassium) inside the cytoplasm instead of synthesizing organic osmolytes so as to maintain the ionic concentration in the cell equivalent or higher than the external environment (Jensen et al. 2015). As the 'salt- in strategy' is common in extreme haloarchaea, occurrence of this strategy in simulated microgravity was studied by detecting the intracellular archaeal accumulation of ions by $H$. argentinensis RR10 using EDAX analysis. In the current study, $H$. argentinensis RR10 demonstrated greater sequestration of chloride followed by sodium ions in NG as compared to SMG conditions. Presence of a light independent $\mathrm{Cl}^{-}$transport channel (Nad and Nac complex) has been identified in the genome of Haloarcula marismortui. Similarly, haloarchaea possess halorhodopsin which is a light gated ion channel or a light driven chloride pump that is causal for chloride accumulation inside the cell (Jensen et al. 2015). It is known that the archaeon $H$. argentinensis has presence of retinal proteins in the membrane (Ihara et al. 1997). The isolate may thus use halorhodopsin or the light independent $\mathrm{Na}^{+} / \mathrm{Cl}^{-}$symporter or transporter to sequester chloride ions in cytoplasm during its growth in normal gravity of $1 \mathrm{~g}$. The chloride anions may possibly react with the hydroxyl radicals in the cell to generate lesser reactive chloride radicals that tend to mitigate the deleterious effects of oxidative damage caused by more reactive hydroxyl radicals. Generally, haloarchaea increase the intracellular influx of potassium ions instead of sodium ions, driven by proton motive force via a Trk $\mathrm{K}^{+} / \mathrm{H}^{+}$ symport while growth in osmotic stress (Jensen et al. 2015). However, in H. argentinensis RR10, the sequestration of sodium ions is higher as compared to potassium ions. This may be due to the plausible preferential sequestration of sodium ions and replacement of potassium ion when exposed to SMG.

The study of differential proteins expression was studied in $H$. argentinensis. The differences in the bands present in the protein fraction of the isolate incubated in SMG and NG was apparent. Previous studies have reported a notable difference in protein profile when $H$. mediterranei DSM $1411^{\mathrm{T}}$ and Halococcus dombrowskii DSM $14522^{\mathrm{T}}$ were exposed to SMG in exponential phase (Dornmayr-Pfaffenhuemer et al. 2011). However, the proteins expressed in haloarchaea in response to simulated microgravity were not identified previously (DornmayrPfaffenhuemer et al. 2011). Moreover, when the proteins are separated using 2-D gel electrophoresis, the majority of haloarchaeal proteins being acidic, aggregate and cluster as they are in a narrow pI range (4.2-5.2) causing difficulty in separation and study of these proteins (Thombre et al. 2016b). To avoid these problems in the present study, we directly excised the differential protein band expressed in simulated microgravity conditions in 1-D SDS-PAGE gels and trypsinized them and attempted to identify the peptide mass fingerprint generated using MALDI-TOF MS (Trotter et al. 2015). The major peptide samples analyzed and identified by MASCOT revealed the presence of $50 \mathrm{~S}$ ribosomal protein L22, 50S ribosomal protein L31e, 30S ribosomal protein S8, 30S ribosomal protein S11and Translation initiation factor 2 subunit-betain $H$. argentinensis. Studies related to the proteome analysis of cells exposed to SMG are limited. The proteomic analysis of Daphnia exposed to SMG demonstrated upregulation of proteins related to actin microfilament organization, protein folding and energy metabolism-related proteins (Trotter et al. 2015). The genomic studies related to haloarchaea reveal that genes encoding for functional classes of chaperones (CHP), signal transduction (SIG) and cellular processes (CP) are up regulated during exponential phase as compared to stationary phase (Lange et al. 2007). The changes in environmental conditions leads to stress which is the cardinal causal factor responsible for upregulation of chaperonins, proteasomal proteins and small heat shock proteins (sHSP's) in haloarchaea (Thombre and Oke 2015; Thombre et al. 2016b). The genomic data of E. coli K12 MG1655(cultured in microgravity under clinorotation) analyzed based on the DAVID geneontology (GO) term enrichments revealed that genes for DNA transcription, helicases, multidrug efflux system and small RNA were upregulated and genes involving stress proteins were downregulated (Arunasri et al. 2013). A global response to simulated microgravity involving a general regulator of transcription has been envisaged in bacteria (Wilson et al. 2007).In the present study, the maximum proteins differentially expressed were ribosomal proteins indicating activation transcription process. This indicates that microgravity does not hamper growth of the organisms, on the contrary the archaeon continues to demonstrate active transcription process. 
Haloarchaea are extremely resistant to antibiotics and when cells are exposed to microgravity, their antibiotic resistance increases markedly (Dornmayr-Pfaffenhuemer et al. 2011). When $H$. argentinensis was exposed to SMG, it altered the resistance pattern of the organism and it developed resistance to many antibiotics like Augmentin, Norfloxacin, Tobramycin and Cefoperazone in addition to its resistance to Nalidixic acid. The antibiotic susceptibilities of the organism are altered during microgravity and analogous findings of increase in susceptibility to antibiotic flucanozole by $C$. albicans in response to SMG stress has been reported (Jiang et al. 2014). Currently the data regarding drug resistance and break points as per Clinical Laboratory Standards Institute (CLSI) and European Committee on Antimicrobial Susceptibility Testing (EUCAST) for classification of multi drug resistance in haloarchaea is not available. As per CLSI and EUCAST an organism is considered multi drug resistant if it develops resistance to at least one agent in three or more antimicrobial categories (Mapara et al. 2015; Shinde and Thombre 2016). Since $H$. argentinensis RR10 developed resistance to at least one agent in three or more antimicrobial categories (viz. Aminoglycoside, Quinolone, Fluoroquinolone, Cephalosporins and Penicillin) it can be inferred that the haloarchaeon develops multi drug resistance on exposure to simulated microgravity. Exposure to simulated microgravity thus increases the antibiotic resistance and renders the culture as Multi-Drug Resistant (MDR) strain of $H$. argentinensis RR10.

Microbial mechanisms of resistance to antibiotics are plasmid encoded resistance, cell wall alterations, and changes in permeability of membranes, production of antibiotic inactivating enzymes and activation of drug efflux pumps (Mapara et al. 2015). Multidrug resistance in microorganisms has garnered impending importance in public health during recent times due to its inherent role in causing life threatening diseases and outbreaks. The antibiotic resistance of haloarchaea is thus of paramount significance as its role in pathogenesis and etiology in human disease is still largely unknown. Besides, considering that these organisms are used to study astrobiology and as exposure to microgravity increases their pathogenicity and antimicrobial resistance (Wilson et al. 2007) further studies related to the mechanisms of drug resistance in these haloarchaea is imperative. Studies related to the presence of antibiotic efflux pumps in haloarchaea are meagre. Antibiotic efflux pump has been detected only in Haloferax volcanii that mediates the transport of doxorubicin, vinblastin, vincristin, ethidium and monensin (Miyauchi et al. 1992). In the present study, we have reported the presence of antibiotic efflux pump in $H$. argentinensis RR10 using the modified ethidium bromide cartwheel assay for the first time. The increase in pigmentation due to synthesis of carotenoid was also notable in haloarchaea exposed to SMG. These carotenoid pigments are involved in cellular protection of the haloarchaea against oxidative damage caused by deleterious effects of stress.

\section{Conclusion}

The present study is presumably the first report on studying the growth kinetics and physiological response of $H$. argentinensis RR10 in simulated microgravity in a clinostat. It is difficult to explain how the mechanical forces of fluid shear transmits intracellular signals to microbial cells at molecular level. The results of the present study indicate that when $H$. argentinensis RR10 is exposed to simulated microgravity, it predominantly responds by acceleration of growth, increase in cellular pigmentation, activation of 'salt-in' strategy of osmoadaptation and upregulation of transcription related ribosomal proteins. Simulated microgravity also causes the increase in antibiotic resistance of the organism and upregulation of antibiotic efflux pumps. The results suggest that there may be a possibility of cross talk between microbial signal transduction systems and future studies might reveal common mechanotransduction themes between these systems and those used to sense and respond to low-shear stress and changes in gravitation forces (Nickerson et al. 2004).

Acknowledgements This research was financially supported by Indian Space Research Organization (ISRO)-Space Technology CellUniversity of Pune (STC-UoP), Grant No. 140, India. We thank Principal, Modern College, Shivajinagar, Pune for providing necessary facilities for the work. Advanced Centre for Treatment, Research and Education in Cancer (ACTREC, Mumbai, India) and IISER, Pune are acknowledged for MALDI-TOF MS analysis and fluorescence microscopy studies. We would like to acknowledge and thank the Department of Physics, Savitribai Phule Pune University for providing the simulated microgravity (Clinostat) facility.

\section{Compliance with ethical standards}

Competing interest The authors declare no conflict of interest.

Open Access This article is distributed under the terms of the Creative Commons Attribution 4.0 International License (http:// creativecommons.org/licenses/by/4.0/), which permits unrestricted use, distribution, and reproduction in any medium, provided you give appropriate credit to the original author(s) and the source, provide a link to the Creative Commons license, and indicate if changes were made.

\section{References}

Abbes M, Baati H, Guermazi S, Messina C, Santulli A, Gharsallah N (2013) Biological properties of carotenoids extracted from 
Halobacterium halobium isolated from a Tunisian solar saltern. BMC Complement Altern Med 13:255-262

Arunasri K, Adil M, VenuCharan K, Suvro C, Himabindu RS, Shivaji S (2013) Effect of simulated microgravity on E. coli K12 MG1655 growth and gene expression. PLoS One 8(3):e57860. doi:10.1371/journal.pone.0057860

Baranyi J, Roberts TA (1994) A dynamic approach to predicting bacterial growth in food. Int J Food Microbiol 23:277-294

Brown RB, Klaus D, Todd P (2002) Effects of space flight, clinorotation and centrifugation on the substrate utilization efficiency of $E$. coli. Micro Sci Tec 13:24-29

Clinical Laboratory Standards Institute (2012) Performance standards for antimicrobial disc susceptibility tests, Twenty second Informational supplement, Tech. Rep. M100-S22, CLSI. Wayne, $\mathrm{Pa}$, USA, vol 32, pp 50-60

Clinical Laboratory Standards Institute (2014) Performance standards for antimicrobial disc susceptibility tests, Twenty fourth Informational supplement, Tech. Rep. M100-S24, CLSI. Wayne, Pa, USA, vol 34, pp 1-230

DasSarma S (2006) Extreme halophiles are models for astrobiology. Microbe 1(3):120-126

Dornmayr-Pfaffenhuemer M, Legat A, Schwimbersky K, Fendrihan S, Stan-Lotter H (2011) Response of Haloarchaea to simulated microgravity. Astrobiology 11:199-205

Fendrihan S, Legat A, Pfaffenhuemer M, Gruber C, Weidler G, Gerbl F (2006) Extremely halophilic archaea and the issue of long-term microbial survival. Rev Environ Sci Biotechnol 15:203-218

Govekar R, Kawle K, Thomas R, Advani S, Sheena PV, Zingde S (2012) Eryptotic phenotype in chronic myeloid leukemia: contribution of neutrophilic cathepsin G. Anemia. doi:10.1155/ 2012/659303

Ihara K, Watanabe S, Tamura T (1997) Haloarcula argentinensis sp. nov. and Haloarcula mukohataei sp. nov., two new extremely halophilic archaea collected in Argentina. Int J Syst Bacteriol 47:73-77

Jagtap SS, Awhad RB, Santosh B, Vidyasagar PB (2011) Effects of clinorotation on growth and chlorophyll content of rice seeds. Microgravity Sci Technol 23:41-48

Jensen M, Matlock S, Reinheimer C, Lawlor C, Reinheimer T, Gorrell A (2015) Potassium stress growth characteristics and energetics in the haloarchaeon Haloarcula marismortui. Extremophiles 19:315-325

Jiang W, Xu B, Yi Y, Huang Y, Li XO, Jiang F (2014) Effects of simulated micro-gravity by RCCS on the biological features of Candida albicans. Int J Clin Exp Pathol 7:3781-3790

Klaus D, Simske S, Todd P, Stodieck L (1997) Investigation of space flight effects on Escherichia coli and proposed model of underlying physical mechanisms. Microbiology 143:449-455

Klaus DM, Todd P, Schatz A (1998) Functional weightlessness during clinorotation of cells suspensions. Adv Space Res $21: 1315-1318$

Lange C, Zaigler A, Hammelmann M, Twellmeyer J, Raddatz G, Schuster SC (2007) Genome-wide analysis of growth phasedependent translational and transcriptional regulation in halophilic archaea. BMC Genom 8:415. doi:10.1186/1471-2164-8415

Luna C, Yew AG, Adam HH (2015) Effects of angular frequency during clinorotation on mesenchymal stem cell morphology and migration. Npj Microgravity 1:15007. doi:10.1038/npjmgrav. 2015.7

Mancinelli R, White MR, Rothschild LJ (1998) Biopan survival I: exposure of the osmophiles Synechococcus sp. (Nägeli) and Haloarcula sp. to the space environment. Adv Space Res 22:327-334

Mapara N, Sharma M, Shriram V, Bharadwaj R, Mohite KC, Kumar $\mathrm{V}$ (2015) Antimicrobial potentials of Helicteres isora silver nanoparticles against extensively drug-resistant (XDR) clinical isolates of Pseudomonas aeruginosa. Appl Microbiol Biotechnol. doi:10.1007/s00253-015-6938-x

Martins M, Viveiros M, Couto I, Costa SS, Pacheco T, Fanning S (2011) Identification of efflux pump-mediated multidrug-resistant bacteria by the Ethidium bromide-agar Cartwheel method. In vivo $25: 171-178$

Metris A, George SM, Baranyi J (2006) Use of optical density detection times to assess the effect of acetic acid on single-cell kinetics. Appl Environ Microbiol 72(10):6674-6679

Miyauchi S, Komatsubara M, Kamo N (1992) In archaeabacteria, there is a doxorubicin efflux pump similar to mammalian P-glycoprotein. Biochem. Biophys. Acta. 1110:144-150

Nickerson CA, Ott CM, Wilson JW, Ramamurthy R, Pierson DL (2004) Microbial responses to microgravity and other low-shear environments. Microbiol Mol Biol Rev 68:345-361

Otte K, Frohlich T, Arnold G, Laforsh C (2014) Proteomic analysis of Daphnia magna hints at molecular pathways involved in defensive plastic responses. BMC Genom 15:306. doi:10.1186/ 1471-2164-15-306

Purevdorj-Gage B, Sheehan KB, Hyman LE (2006) Effects of lowshear modeled microgravity on cell function, gene expression, and phenotype in Saccharomyces cerevisiae. Appl Environ Microbiol 72:4569-4575

Rieger AM, Nelson KL, Konowalchuk JD, Barreda DR (2011) Modified annexin V/propidium iodide apoptosis assay for accurate assessment of cell death. JoVE 50:2597. doi:10.3791/ 2597

Robinson JL, Pyzyna B, Atrasz RG, Henderson CA, Morrill KL, Burd AM (2005) Growth kinetics of extremely halophilic Archaea (Family Halobacteriaceae) as revealed by Arrhenius plots. J Bacteriol 187(3):923-929

Salgaonkar B, Das D, Braganca J (2016) Resistance of extremely halophilic archaea to zinc and zinc oxide nanoparticles. Appl Nanosci 6:251-258

Sehgal SN, Gibbons NE (1960) Effect of some metal ions on the growth of Halobacterium cutirubrum. Can J Microbiol $6: 165-169$

Shinde V, Thombre RS (2016) Antibiotic resistance profiling of marine halophilic bacteria and haloarchaea. J App Pharm Sci 6(10):132-137

Shivanand P, Mugeraya G (2011) Halophilic bacteria and their compatible solutes-osmoregulation and potential applications. Curr Sci 100:1516-1521

Stan-Lotter H, Pfaffenhuemer M, Legat A, Busse HJ, Radax C, Gruber C (2002) Halococcus dombrowskii sp. nov., an archaeal isolate from a Permian alpine salt deposit. Int J Syst Evol Microbiol 52:1807-1814

Thombre RS, Oke RS (2015) Study of stress proteins induced by temperature stress in extremely halophilic archaea, Haloferax mediterranei RT18. Int $\mathrm{J}$ Curr Microbiol App Sci 2:199-209

Thombre R, Joshi V, Oke R (2016a) Halophiles: pharmaceutical potential and biotechnological applications. In: Thangadurai D, Jeyabalan S (eds) Industrial biotechnology: sustainable production and bioresource utilization. Apple Academic Press Inc., Waretown, pp 111-139

Thombre RS, Shinde V, Oke R, Dhar SK, Shouche YS (2016b) Biology and survival of extremely halophilic archaeon Haloarcula marismortui RR12 isolated from Mumbai salterns, India in response to salinity stress. Sci Rep 6:25642. doi:10.1038/ srep 25642

Thombre R, Shinde V, Thaiparambil E, Zende S, Mehta S (2016c) Antimicrobial activity and mechanism of inhibition of silver nanoparticles against extreme halophilic archaea. Front Microbiol 7:1424. doi:10.3389/fmicb.2016.01424 
Thombre R, Bhalerao A, Shinde V, Dhar S, Shouche Y (2017) Response of archaeon Natronococcus jeotgali RR17 to hypergravity. Microgravity Sci Technol. doi:10.1007/s12217-0179538-9

Trotter B, Otte K, Schoppmann K, Hemmersbach R, Fröhlich T, Arnold G (2015) The influence of simulated microgravity on the proteome of Daphnia magna. npj Microgravity 1:15016. doi:10. 1038/npjmgrav.2015.16

Wilson JW, Ott CM, HönerzuBentrup K, Ramamurthy R, Quickm L, Porwollik S (2007) Space flight alters bacterial gene expression and virulence and reveals a role for global regulator Hfq. PNAS 104:16299-16304

Xu B, Li C, Zheng Y, Si S, Hunag Y, Zhang J, Cui Y (2015) Simulated microgravity affects ciprofloxacin susceptibility and expression of acrAB-tolC genes in E. coliATCC25922. Int J Clin Exp Pathol 8:7945-7952

Yamazaki T, Yoshimoto M, Nishiyama Y, Okubo Y, Makimura K (2012) Phenotypic characterization of Aspergillus niger and Candida albicans grown under simulated microgravity using a three-dimensional clinostat. Microbiol Immunol 56:441-446 